\title{
G117-B15A: HOW IS IT EVOLVING?
}

\author{
S.O.Kepler \\ Instituto de Fisica \\ Universidade Federal do Rio Grande do Sul \\ Brazil, \\ G.Vauclair \\ Observatoire Midi-Pyrenees \\ France, \\ and \\ R.E.Nather, D.E.Winget, and E.L.Robinson \\ McDonald Observatory and Department of Astronomy \\ The University of Texas at Austin \\ U.S.A.
}

\begin{abstract}
The measurement of the rate of change of period with time for the g-mode pulsations in ZZ Ceti stars is a direct measurement of the cooling timescale for a DA white dwarf, which in turn can give a totally independent measurement of the age of the galactic disk. Using asteroseismology, we have obtained a rate of change of the period of the dominant pulsation in the light curve of the ZZ Ceti star G117-B15A of $d P / d t=(12.5 \pm 5.5) \times 10^{-15} \mathrm{~s} / \mathrm{s}$, equivalent to a timescale for period change of $P / \dot{P}=(5.5 \pm 2.4) \times 10^{8} \mathrm{yr}$, which is consistent with the theoretical value for the cooling timescale of a DA white dwarf around $11,000 \mathrm{~K}$.
\end{abstract}

\section{INTRODUCTION}

The ZZ Ceti stars are the single normal DA white dwarfs which show luminosity variations. Fontaine et. al. (1982) and Greenstein (1982), using a homogeneous set of colors, found that all observed DA white dwarfs in the color range corresponding to effective temperatures in a narrow strip ( $\sim 1400 \mathrm{~K}$ wide) around $11000 \mathrm{~K}$ were $\mathrm{ZZ} \mathrm{Ceti} \mathrm{stars.} \mathrm{Similar} \mathrm{results} \mathrm{were} \mathrm{obtained} \mathrm{from} \mathrm{IUE}$ spectra (Wesemael, Lamontagne and Fontaine 1986). There are 20 known ZZ Cetis, all multiperiodic, with periods ranging from 109 to $1186 \mathrm{sec}$ and peak-to- peak light variations from 2 to $34 \%$. The observed variations are due to nonradial g-mode pulsations (Kep.er 1984) and, since each periodicity constrain independently the properties and structure of the star, the observed pulsations can ideally be used to infer the whole structure of the white dwarf, as demonstrated by the mode trapping calculations of Winget et. al. (1982). For a review of the properties of the ZZ Ceti stars, see Winget (1988). The timescale for period change for the pulsations observed in the ZZ Ceti stars is directly proportional to the cooling timescale of the white dwarf, which can be transformed to the white dwarf birth rate through the measurement of their space densities. The rate of period change also gives directly the core composition, since the cooling timescale is directly related to the equation of state of the white dwarf core and can be used to distinguish among different core-composition white dwarfs (Robinson and Kepler 1980, Winget and Van Horn 1987). Most importantly, the white dwarf cooling timescale gives a totally independent measurement of the age of the galactic disk (Winget et al. 1987).

Continuing our work reported in Kepler et. al. (1982), we have observed one the simplest ZZ Ceti stars, G117-B15A, with high speed photometry to measure its rate of period change. 
Table 1

Journal of Observations since 1982

\begin{tabular}{|c|c|c|c|c|c|}
\hline$\overline{\text { Run }}$ & $\begin{array}{l}\text { Length of } \\
\text { Observation } \\
(\mathrm{hr})\end{array}$ & $\begin{array}{l}\text { Integration } \\
\text { Time (sec) }\end{array}$ & $\begin{array}{l}\text { Fractional } \\
\text { Semi- } \\
\text { Amplitude } \\
\end{array}$ & $\begin{array}{l}\text { BJDD at Maximum } \\
(2440000.0+)\end{array}$ & $\begin{array}{r}\text { Error } \\
(\mathrm{sec})\end{array}$ \\
\hline 2593 & 0.9 & 10 & 0.024 & 4637.776174 & 1.6 \\
\hline 2597 & 7.0 & 10 & 0.023 & 4641.624287 & 0.5 \\
\hline 2629 & 2.1 & 10 & 0.022 & 4992.789531 & 1.2 \\
\hline 2633 & 6.6 & 10 & 0.021 & 4994.689956 & 0.7 \\
\hline 2637 & 3.4 & 10 & 0.024 & 4996.744801 & 0.8 \\
\hline 2640 & 3.7 & 10 & 0.024 & 4997.723649 & 0.7 \\
\hline JES1 & 2.9 & 10 & 0.021 & 5021.716661 & 1.0 \\
\hline 2869 & 3.2 & 10 & 0.022 & 5703.860004 & 1.4 \\
\hline 2870 & 5.6 & 10 & 0.028 & 5734.642701 & 0.7 \\
\hline 2872 & 2.4 & 10 & 0.025 & 5735.643972 & 0.8 \\
\hline ER60 & 3.8 & 5 & 0.023 & 6113.763716 & 0.7 \\
\hline ER110 & 1.8 & 5 & 0.021 & 6443.775386 & 1.0 \\
\hline 3096 & 5.9 & 5 & 0.023 & 6468.630178 & 0.5 \\
\hline 3106 & 1.2 & 10 & 0.024 & 6473.718679 & 0.9 \\
\hline 3141 & 1.0 & 10 & 0.022 & 6523.620086 & 1.3 \\
\hline 3143 & 1.0 & 10 & 0.020 & 6524.613917 & 1.3 \\
\hline AT13* & 3.8 & 5 & 0.018 & 6768.855451 & 2.3 \\
\hline 9004 & 2.0 & 10 & 0.022 & 6794.935676 & 1.0 \\
\hline 9009 & 1.2 & 10 & 0.018 & 6796.928219 & 1.9 \\
\hline 9012 & 1.9 & 10 & 0.022 & 6797.924535 & 1.3 \\
\hline 9014 & 2.8 & 10 & 0.024 & 6798.903378 & 0.9 \\
\hline DEW1 & 1.7 & 10 & 0.018 & 6823.663537 & 1.5 \\
\hline DEW2 & 4.5 & 10 & 0.020 & 6825.651132 & 1.6 \\
\hline GV1 & 2.4 & 6 & 0.022 & 7231.328096 & 1.1 \\
\hline REN23 & 3.0 & 10 & 0.022 & 7231.612054 & 0.7 \\
\hline GV2 & 1.5 & 6 & 0.017 & 7232.396626 & 3.4 \\
\hline REN25 & 2.4 & 10 & 0.020 & 7232.623291 & 2.7 \\
\hline GV3 & 2.9 & 6 & 0.019 & 7233.343090 & 2.1 \\
\hline REN27 & 2.2 & 10 & 0.022 & 7233.634506 & 1.9 \\
\hline GV4 & 3.4 & 6 & 0.022 & 7234.319475 & 1.3 \\
\hline GV5 & 3.2 & 6 & 0.020 & 7235.313250 & 1.6 \\
\hline REN30 & 2.7 & 10 & 0.021 & 7235.607168 & 1.2 \\
\hline REN32 & 2.5 & 10 & 0.022 & 7236.610922 & 0.9 \\
\hline
\end{tabular}

* data taken on the $91 \mathrm{~cm}$ telescope, with companion inside aperture

\section{OBSERVATIONS}

In 1982 we used our 85 hours of high speed photometry on the star to show that the light curve of G117-B15A has at least six pulsation modes simultaneously excited with periods of $107.6 \mathrm{~s}$, $119.8 \mathrm{~s}, 126.2 \mathrm{~s}, 215.2 \mathrm{~s}, 271.0 \mathrm{~s}$, and $304.4 \mathrm{~s}$. The $215.2 \mathrm{~s}$ pulsation has a fractional semiamplitude of 0.022 , nearly 3 times greater than that of any other pulsation and dominates completely the light curve. We could then derive an upper limit for the rate of change of period with time of $|\dot{P}| \leq 7.8 \times 10^{-14} \mathrm{~s} / \mathrm{s}$ for this pulsation.

We have since then obtained additional 80 hours of high speed photometry using a two-star photometer (Nather 1973) on the $2.1 \mathrm{~m}$ Struve telescope, plus 4 hours on the $91 \mathrm{~cm}$ telescope, at 
McDonald Observatory, plus another 13 hours on the $1.9 \mathrm{~m}$ telescope at Haute Provence Observatory to improve our estimate of the rate of period change for this pulsation. The data now spans 13 years. The observations were obtained with a blue-sensitive bialkali phototube, and as G117-B15A is rather faint, $V=15.52$ (Eggen and Greenstein 1965), all measurements were obtained in unfiltered light in order to improve the photon detection rate. The amplitudes observed should be used with caution, since they are wavelength dependent (Robinson, Kepler, and Nather 1982, Brassard, Wesemael and Fontaine 1987), and the effective wavelength of our white-light photometry is not well determined. Table 1 gives a journal of the observations since 1982. The quoted errors throughout this paper are one standard deviation.

\section{DATA ANALYSIS}

The method for data reduction and conversion of the time base from UTC to the more uniform Barycentric Julian Dynamical Date (prior Barycentric Julian Ephemeris Date) was described in Kepler et. al. (1982).

The first step of our analysis was to take Fourier transforms of each night's data to verify which pulsations were present. We had to take in account that only on the runs longer than about 4 hours we could see the three largest amplitude pulsations separetely, and that the three smallest amplitude pulsations could only be seen in power spectra taken with all the seasonal data at once. Our conclusion is that the pulsational power spectrum of G117-B15A has remained the same during the 13 years we have observed the star; all the 6 pulsations detected in 1982 are still present in the data, with the $215.2 \mathrm{~s}$ pulsation dominating the light curve.

4. THE UPPER LIMIT TO $\dot{P}$

Our method of measuring the rate of period change is as follows: first, we fit a sinusoidal with a period of $215.2 \mathrm{~s}$ to the data by least squares to obtain the time of maximum of the pulsation for each night's data. After converting the obtained time of maxima to the barycenter of the solar system, we calculate the difference between the observed $(O)$ times of maxima and the times of maxima calculated $(\mathrm{C})$ using a linear ephemeris starting on our first observation, obtaining therefore an (OC) diagram (Figure 1). Unique cycle counts $E$ were obtained for each night since the period derived in 1982 was extremely accurate, permitting us to bridge the whole data base without any possibility of cycle count error. The fairly large apparent scatter in the diagram is an artifact of the varying quality of the different runs; no data point lies more than $3 \sigma$ away from zero.

The rate of period change for the pulsation, as well as a correction to the period and phase, can then be obtained by fitting a parabola to the $(\mathrm{O}-\mathrm{C})$, since:

$$
(O-C)=E_{0}+P \cdot E+\frac{1}{2} P \cdot \dot{P} \cdot E^{2}
$$

where $E_{0}$ is the epoch of observation, $P$ is the period, $E$ is the number of cycles elapsed since $E_{0}$, and $\dot{P}$ is the rate of change of period with time, $d P / d t$.

Using this formulae to fit a parabola by weighted least-squares to the 69 times of maxima obtained since 1975, one for each run of data, we obtain:

$$
\begin{aligned}
E_{0}= & \text { BJDD 2442397.917521 } \pm 0.000007 \text { days } \\
& P=215.1973870 \pm 0.0000013 \mathrm{sec} \\
& d P / d t=(12.5 \pm 5.5) \times 10^{-15} \mathrm{~s} / \mathrm{s}
\end{aligned}
$$

or

$$
P / \dot{P}=(5.5 \pm 2.4) \times 10^{8} \mathrm{yr}
$$

The observed $\dot{P}$ should be considered only as an upper limit, since our measurement of it is still not converging to any value, as can be seen from Table 2, which gives the result of the parabola fit to the $(\mathrm{O}-\mathrm{C})$ including data obtained from 1975 up to that year. 
Table 2

Evolution of the $P$ Measurement

\begin{tabular}{cc}
\hline Year & $d P / d t$ \\
\hline 1980 & $(5.5 \pm 4.7) \times 10^{-14}$ \\
1981 & $(5.3 \pm 2.3) \times 10^{-14}$ \\
1982 & $(0.4 \pm 2.2) \times 10^{-14}$ \\
1984 & $(0.5 \pm 1.3) \times 10^{-14}$ \\
1985 & $(0.4 \pm 1.1) \times 10^{-14}$ \\
1986 & $-(0.6 \pm 0.8) \times 10^{-14}$ \\
1987 & $-(0.4 \pm 0.7) \times 10^{-14}$ \\
1988 & $(1.2 \pm 0.6) \times 10^{-14}$ \\
\hline
\end{tabular}

\section{DISCUSSION}

The observational upper limit on the rate of period change can be compared with the theoretical rate of period change of nonradial g- mode pulsations calculated by Winget (1981), Wood and Winget (1988), and Bradley, Winget and Wood (1988 - these Proceedings), caused by the cooling of the white dwarf. The calculations were made with linear, nonadiabatic pulsation code, and used evolutionary models for carbon core white dwarfs incorporating stratified $\mathrm{H} / \mathrm{He}$ envelopes consistent with diffusion equilibrium. For models corresponding to the $\mathrm{ZZ}$ Ceti stars, they found $\dot{P} \approx 3 \times 10^{-15}$ $\mathrm{s} / \mathrm{s}\left(P / \dot{P} \approx 2 \times 10^{9} \mathrm{yr}\right)$ for a pure carbon core white dwarf and modes with low $k$ and $\ell$ values, at a temperature of $T_{\text {eff }} \approx 13000$ like G117-B15A. As demonstrated by Dziembowski (1977), simple geometrical considerations imply that the observed modes are low $\ell$ values. Since the heat leakage is proportional to the mean atomic weight of the core composition, the rate of change of period with time for a white dwarf with $\mathrm{Fe}$ core can be as large as $\dot{P} \approx 1.4 \times 10^{-14} \mathrm{~s} / \mathrm{s}\left(P / \dot{P} \approx 5 \times 10^{8} \mathrm{yr}\right)$, for low $k$ and $\ell$ g-modes. Our results are therefore still consistent with the theoretical expectations for $g$-modes, even for models with an Fe-core.

Since our limit on $P / \dot{P}$ will continue to improve quadratically with the time baseline the star is observed, or about $17 \%$ per year nowadays, we expect to reach the precision predicted time scale for a $\mathrm{C} / \mathrm{O}$ core in 10 years. If, on the other hand, the value for $\dot{P}$ we obtained is real, in 2 years it will be a $3 \sigma$ measurement. We can therefore measure the evolutionary timescale for a cool white dwarf, equivalent to that measured by Winget et al. (1985) for the hot white dwarf PG1159-035. These measurements are crucial to an independent way of directly measuring the age of the galactic disk, which in turn can be used to measure the age of the Universe.

In order to measure a rate of change of period with time of the order of $10^{-14} \mathrm{~s} / \mathrm{s}$, like the one observed in G117-B15A, there is a very stringent restriction on the dynamical environment of the white dwarf, since even a planet of the mass of Jupiter, and at the same distance from the white dwarf that Jupiter is from the Sun, would give an apparent rate of change of the pulsation period, i.e., of the arrival time of the pulses of the order of $10^{-12} \mathrm{~s} / \mathrm{s}$, with the times of maxima varying periodically as the pulsating star moves around the barycenter of the system. That means that to give a $\dot{P}$ of the order of $10^{-14} \mathrm{~s} / \mathrm{s}$, the white dwarf cannot have any body of even a large planet mass orbiting it. The technique of measuring rates of change of periods with time may therefore prove itself ultimately capable of detecting planets outside the solar system, in addition to producing information on the stellar structure and evolution.

This work was partially supported by grants from CNPq-Brazil, NSF-USA, and CNRS-France.

\section{REFERENCES}

Brassard, P., Wesemael, F., and Fontaine, G. 1987, in Proceedings of the IAU Colloquium 95, The Second Conference on Faint Blue Stars, ed. A.G.D.Philip, D.S.Hayes, and J.W.Liebert, (L.Davis Press, Schenectady), p. 669.

Dziembowski, W. 1977, Acta Astron., 27, 203. 
Eggen, O.J., and Greenstein, J.L. 1965, Ap. J., 141, 183.

Fontaine, G., McGraw, J.T., Dearborn, D.S.P., Gustafson, J., and Lacombe, P. 1982, Ap. J., 258, 651.

Greenstein, J.L. 1982, Ap. J., 258, 661.

Kepler, S.O. 1984, Ap. J., 286, 314.

Kepler, S.O., Robinson, E.L., Nather, R.E., and McGraw, J.T. 1982, Ap. J., 254, 676.

Nather, R.E. 1973, Vistas in Astronomy, 15, 91.

Robinson, E.L., and Kepler, S.O. 1980, Spa. Sci. Rev., 27, 613.

Robinson, E.L., Kepler, S.O., and Nather, R.E. 1982, Ap. J., 259, 219.

Wesemael, F., Lamontagne, R., and Fontaine, G. 1986, Astron. J., 91, 1376.

Winget, D.E. 1981, Ph.D. Thesis, University of Rochester.

Winget, D.E. 1988, in Proceedings of IA U Symposium 123, Advances in Helio- and Asteroseismology, ed. J.Cristensen-Dalsgaard and S. Frandsen, (D.Reidel, Dordrecht), p. 305.

Winget, D.E., Hansen, C.J., Liebert, J., Van Horn, H.M., Fontaine, G., Nather, R.E.,Kepler, S.O., and Lamb, D.Q. 1987, Ap. J., 315, L77.

Winget, D.E., Kepler, S.O., Robinson, E.L., Nather. R.E., and O'Donoghue, D. 1985, Ap. J., 292, 606

Winget, D.E., and Van Horn, H.M. 1987, in Proceedings of the IAU Colloquium 95, The Second Conference on Faint Blue Stars, ed. A.G.D.Philip, D.S.Hayes, and J.W.Liebert, (L.Davis Press, Schenectady), p. 363.

Winget, D.E., Van Horn, H.M., Tassoul, M., Hansen, C.J., Fontaine, G., and Carrol, B.W. 1982, Ap. J., 252, L65.

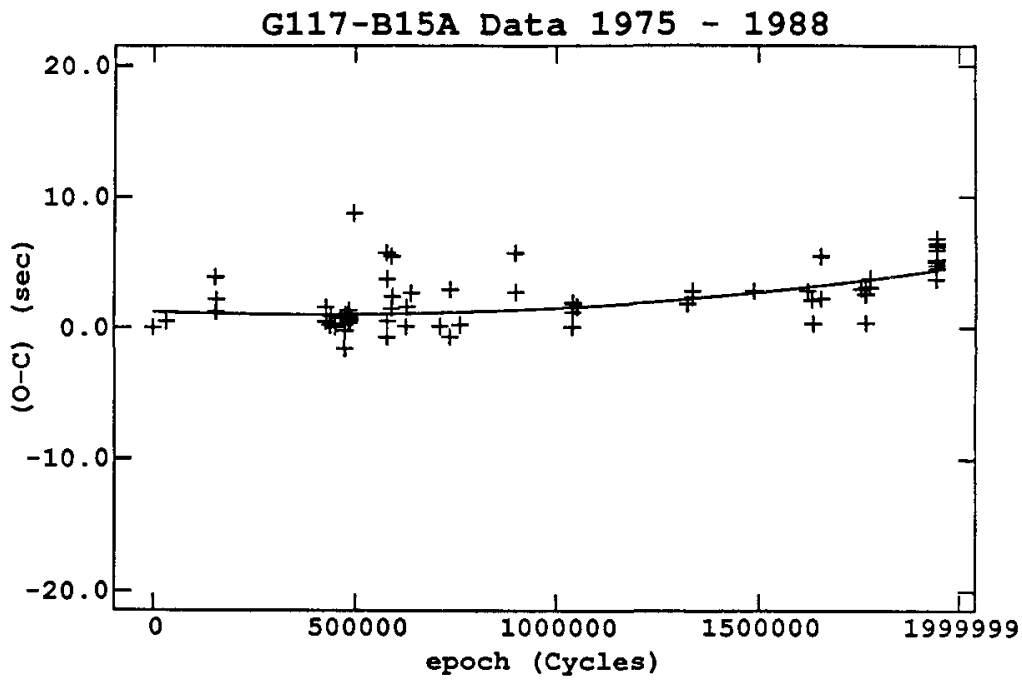

Figure 1. The $(\mathrm{O}-\mathrm{C})$ diagram for the 215.2 s pulsation of G117-B15A. The observed times of maxima are listed in Table 1 , and the calculated times of maxima were derived using a linear ephemeris starting on BJED 2442397.917507. 